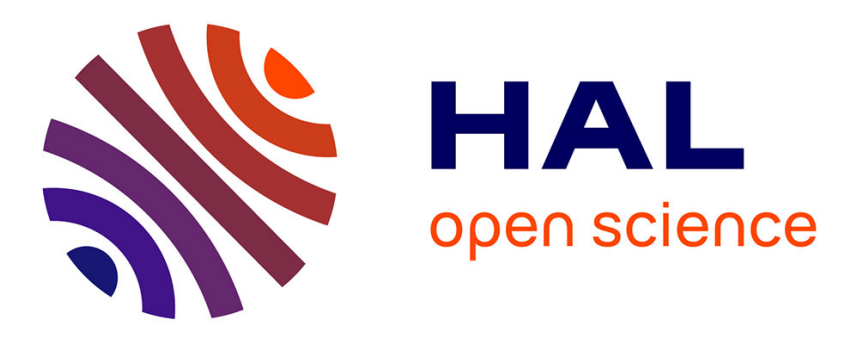

\title{
Forecasting chaotic systems: The role of local Lyapunov exponents
}

\author{
Dominique Guegan, Justin Leroux
}

\section{To cite this version:}

Dominique Guegan, Justin Leroux. Forecasting chaotic systems: The role of local Lyapunov exponents. Chaos, Solitons \& Fractals, 2009, 41 (5), pp.2401-2404. 10.1016/j.chaos.2008.09.017 . halshs00431726v2

\section{HAL Id: halshs-00431726 \\ https://shs.hal.science/halshs-00431726v2}

Submitted on 26 Nov 2009

HAL is a multi-disciplinary open access archive for the deposit and dissemination of scientific research documents, whether they are published or not. The documents may come from teaching and research institutions in France or abroad, or from public or private research centers.
L'archive ouverte pluridisciplinaire HAL, est destinée au dépôt et à la diffusion de documents scientifiques de niveau recherche, publiés ou non, émanant des établissements d'enseignement et de recherche français ou étrangers, des laboratoires publics ou privés. 


\title{
Forecasting chaotic systems:
}

\section{The role of local Lyapunov exponents}

\author{
Dominique Guégan* and Justin Leroux ${ }^{\dagger}$
}

September 5, 2008

\begin{abstract}
We propose a novel methodology for forecasting chaotic systems which is based on exploiting the information conveyed by the local Lyapunov exponents of a system. This information is used to correct for the inevitable bias of most non-parametric predictors. Using simulated data, we show that gains in prediction accuracy can be substantial.
\end{abstract}

*PSE, CES-MSE, Université Paris-I Panthéon Sorbonne, 106 boulevard de l'Hôpital, 75013 Paris, France (Email: Dominique.Guegan@univ-paris1.fr)

${ }^{\dagger}$ Institute for Applied Economics, HEC Montréal and CIRPÉE, 3000 chemin de la CôteSte-Catherine, Montréal, QC H3T 2A7, Canada. (Email: justin.leroux@hec.ca, Fax: (1) 514-340-6469, corresponding author) 


\section{Introduction}

When taking a deterministic approach to predicting the future of a system, the main premise is that future states can be fully inferred from the current state. Hence, deterministic systems should in principle be easy to predict. Yet, some systems can be difficult to forecast accurately: such chaotic systems are extremely sensitive to initial conditions, so that a slight deviation from a trajectory in the state space can lead to dramatic changes in future behavior. We propose a novel methodology for forecasting deterministic series which corrects for the inevitable bias of most non-parametric predictors (such as methods based on kernels, radial functions, neural nets, wavelets, etc.; see [1] and [2]) by incorporating additional information on the local chaoticity of the system via the so-called local Lyapunov exponent (LLE). To the best of our knowledge, while several works exist on the forecasting of chaotic systems (see, e.g., [3], [4], [5], $[6],[7],[8])$, none exploit the information conveyed by the LLE. The general intuition behind the methodology we propose can be viewed as a complement to existing forecasting methods, and can be extended to chaotic time series. For illustrative purposes, we describe how our methodology can be used to improve upon the well-known nearest-neighbor predictor on two deterministic systems.

The nearest-neighbor predictor has proved to be a simple yet useful tool for forecasting chaotic systems (see [9]). In the case of a one-neighbor predictor, it takes the observation in the past which most resembles today's state and returns that observation's successor as a predictor of tomorrow state. The rationale behind this nearest-neighbor predictor is quite simple: given that the system is assumed to be deterministic and ergodic, one obtains a sensible prediction of the variable's future by looking back at its evolution from a similar, past situation. For predictions more than one step ahead, the procedure is iterated by successively merging the predicted values with the observed data. 
The nearest-neighbor predictor performs reasonably well in the short run but is not satisfactory for even medium-run predictions ([10], [11]). The generally accepted intuition being that the two trajectories (of the current state and of its neighbor) will have separated significantly by then, and the nearest neighbor's medium-run future will have little to do with the future we are trying to predict. Intuitively, this failure to perform well in the medium run arises mainly from the fact that short-run predictions are not accurate enough to withstand the complex dynamics of the system and to remain accurate after being iterated over a period of time of significant length. We argue that this lack of accuracy is inherent to the prediction method itself because the nearest neighbor on which predictions are based can never exactly coincide with today's state (or else the underlying process, being deterministic, would also be periodic and, thus, could not be chaotic).

We aim to correct the above shortcoming by incorporating information carried by the system's LLEs into the prediction. The LLE (see [12], [13]) represents the local dispersion rate of the system at a given point: a positive value meaning that two nearby points in the state space tend to grow apart over time, while a negative value indicates that nearby points will come closer together in the near future (but may diverge later on). In other words, the LLE is a measure of local chaoticity of a system. Typically, even a "globally chaotic" system is made up of both "chaotic regions" where the LLE is positive and more stable regions where it is negative. We illustrate this fact, which has been suggested in [14], more systematically in a companion paper.

By definition, the LLE tells us precisely by how much the distance between the current state and its nearest neighbor will expand (or contract) over time, so that we can easily obtain the distance between the nearest-neighbor predictor (i.e., the neighbor's successor) and the future we are trying to predict (tomor- 
row's state). Thus, we know exactly by how much to correct the prediction of the nearest-neighbor predictor. ${ }^{1}$

The rest of the paper is organized as follows. In Section 2, we develop our methodology by first pointing out why the nearest-neighbor predictor information is biased and then suggesting how to correct this bias using information carried by the system's LLEs. In Section 3, we present simulations carried out on known chaotic systems to illustrate the extent of the (large) potential accuracy gains our methodology generates. Finally, Section 4 concludes by discussing the significance of the approach we propose and by pointing to directions in future work in order to refine it.

\section{Methodology}

Consider a one-dimensional series of $T$ observations from a chaotic system, $\left(x_{1}, \ldots x_{T}\right)$, whose future values we are trying to forecast. Recall that a chaotic system is characterized by the existence of an attractor in a $d$-dimensional phase space (see [15]), where $d>1$ is the embedding dimension. ${ }^{2}$ A possible embedding method involves building a $d$-dimensional orbit, $\left(X_{t}\right)$, with $X_{t}=\left(x_{t}, x_{t-\tau}, \ldots, x_{t-(d-1) \tau}\right)$ (see [17]). For the sake of exposition, we shall assume $\tau=1$ in the remainder of the paper.

By definition, the local Lyapunov exponent (or LLE) of a dynamical system which characterizes the rate of separation of infinitesimally close points of an orbit. Quantitatively, two neighboring points in phase space with initial

\footnotetext{
${ }^{1}$ Note that correction is required even in regions of the state space where the system is stable (i.e., where nearby trajectories come closer together, corresponding to a negative value of the LLE).

${ }^{2}$ The choice of the embedding dimension has been the object of much work (see [16] for a survey) and is beyond the scope of this note.
} 
separation $\delta X_{0}$ are separated, $t$ periods later, by the distance:

$$
|\delta X| \approx\left|\delta X_{0}\right| e^{\lambda_{0} t}
$$

where $|\cdot|$ represents the modulus of the considered vectors and $\lambda_{0}$ is the local Lyapunov exponent of the system in the vicinity of the initial points. Typically, this local rate of divergence (or convergence, if $\lambda_{0}<0$ ) depends on the orientation of the initial vector $\delta X_{0}$. Thus, strictly speaking, a whole spectrum of local Lyapunov exponents exists, one per dimension of the state space (see [12]). A dynamic system is considered to be (locally) chaotic if $\lambda_{0}>0$, and (locally) stable if $\lambda_{0}<0$. (see, e.g., [14])

Our goal is to exploit the local information carried by the LLEs to improve upon existing methods of reconstruction and prediction. We propose a methodology which builds upon the classical nearest-neighbor predictor, which we now recall. Consider an orbit $\left(X_{1}, \ldots, X_{T}\right)$ whose one-step-ahead future, $X_{T+1}$, we are trying to predict. The nearest-neighbor predictor returns $\hat{X}_{T+1}=X_{i+1}$, where $X_{i}$ is the element of the orbit with minimal distance to $X_{T}$. Because the dynamic system at hand is aperiodic (or else, forecasting would not be an issue), the nearest-neighbor predictor is inevitably biased. Indeed, because $\left|X_{T}-X_{i}\right|>0$, it must also be the case that:

$$
\left|\hat{X}_{T+1}-X_{i+1}\right| \approx\left|X_{T}-X_{i}\right| e^{\lambda_{i}}>0
$$

where $\lambda_{i}$ can be approximated in practice by the following expression:

$$
\hat{\lambda}_{i}=\ln \frac{\left|X_{i+1}-X_{j+1}\right|}{\left|X_{i}-X_{j}\right|} \quad \text { with } X_{j}=\arg \min _{t \neq i, T}\left|X_{t}-X_{i}\right|
$$

It follows from Expression (1) that knowing the distance between the predictee and the nearest neighbor as well as the LLE at the nearest neighbor allows 
us to predict the distance of the predictee's image to the neighbor's image. Note that this is true regardless of the sign of $\lambda_{i}$; i.e., regardless of whether the system is locally chaotic or locally stable. Moreover, because the orbit considered results from the embedding of a one-dimensional series, we also know all but the first coordinate of $X_{T+1}=\left(x_{T+1}, x_{T}, \ldots, x_{T-d+2}\right)$. Hence, $X_{T+1}$ lies at the intersection of the sphere of radius $\left|X_{T}-X_{i}\right| e^{\hat{\lambda}_{i}}$ centered on $X_{T}$ and the line defined by $\left\{\left(z, x_{T}, \ldots, x_{T-d+2}\right) \mid z \in \mathbb{R}\right\}$ which, in the Euclidean space, amounts to solving the following polynomial for $z \in \mathbb{R}$ :

$$
\left(z-x_{i+1}\right)^{2}+\left(x_{T}-x_{i}\right)^{2}+\ldots+\left(x_{T-d+2}-x_{i-d+2}\right)^{2}-\left|X_{T}-X_{i}\right| e^{\hat{\lambda}_{i}}=0
$$

Typically, two candidates emerge, $\hat{x}_{T+1}^{-}$and $\hat{x}_{T+1}^{+}$, respectively underestimating and overestimating the true value of observation $x_{T+1}$ (see Figure 1$)^{3}$.

\section{[FIGURE 1]}

One difficulty lies in determining when the nearest-neighbor predictor overestimates or underestimates the true value to be predicted. Being able to discriminate accurately between $\hat{x}_{T+1}^{-}$and $\hat{x}_{T+1}^{+}$may significantly improve the accuracy the nearest-neighbor predictor, as we next illustrate.

\section{Simulations}

We illustrate our point by simulating two well-known chaotic systems: the Lorenz system (see [18]) and the logistic map (see [19]). The Lorenz system

\footnotetext{
${ }^{3}$ The situation whereby Expression (3) has no real solution would only arise if $\lambda_{i}$ had been greatly underestimated, which never occurred to us in practice using Expression (2).
} 
is characterized by the following system of differential equations:

$$
\left\{\begin{array}{c}
\frac{d x}{d t}=\sigma(y-x) \\
\frac{d y}{d t}=x(R-z)-y \\
\frac{d z}{d t}=x y-b z
\end{array}\right.
$$

We simulated this system with values $\sigma=16, R=45.92$ and $b=4$, initial values $x_{0}=-10, y_{0}=-10$ and $z_{0}=30$, and a step size of 0.01 . Taking 5,000 observations, deleting the first 1,000 ensure that we are working within the attractor and considering the values on the $x$-coordinate as its own series, we successively predicted the last 1,000 in-sample observations. Each prediction was carried out with the full - and true - information set leading up to it, each time using the best of the two candidates, $\hat{x}_{T+1}^{-}$and $\hat{x}_{T+1}^{+}$(measured in distance to the - known-successor). We obtain results which are always better than with the nearest-neighbor predictor and a mean-squared error which is roughly two-thirds that of the nearest-neighbor predictor. (See Table 1)

\begin{tabular}{|c|c|}
\hline LLE-corrected predictor MSE using best candidate & Nearest-Neighbor Predictor MSE \\
\hline 0.1056 & 0.1552 \\
\hline
\end{tabular}

Table 1: Mean-squared error comparison for the Lorenz system

The logistic map is defined by:

$$
x_{t+1}=4 x_{t}\left(1-x_{t}\right)
$$

Keeping the last 4,500 of 5,000 iterations, embedded in dimension 2, we predicted the in-sample 5,001st observation of 1,000 simulated trajectories with initial values drawn from $\mathrm{U}(0,1)$. We again obtain results which are always bet- 
ter than with the nearest-neighbor predictor and much smaller mean squared errors: of the order of $10^{-11}$ as opposed to $10^{-7}$ with the nearest-neighbor predictor; i.e. the LLE-corrected best-candidate predictor is on average one hundred times more accurate than the traditional nearest-neighbor predictor! (See Table 2)

\begin{tabular}{|c|c|}
\hline LLE-corrected predictor MSE using best candidate & Nearest-Neighbor Predictor MSE \\
\hline $1.5415 \mathrm{e}-011$ & $5.5921 \mathrm{e}-007$ \\
\hline
\end{tabular}

Table 2: Mean-squared error comparison for the logistic map

\section{Concluding comments}

The above preliminary analysis suggests that there is great potential in improving upon the accuracy of the nearest-neighbor predictor by incorporating the information contained in local Lyapunov exponents as in Expression (1). Moreover, such increased precision in short-run prediction may translate into accuracy gains for medium-run predictions, which is currently unsatisfactory with existing techniques. In addition, the general intuition behind the proposed method readily applies to other non-parametric predictors.

Several aspects of the implementation are still to be refined, and will be the object of future work. For instance, consistently discriminating between the two candidates, $\hat{x}_{T+1}^{-}$and $\hat{x}_{T+1}^{+}$, can prove to be a difficult task due to the inherent chaotic nature of the systems at hand. As a first guess, one can select the candidate which maximizes the colinearity between the $X_{i+1}-X_{i}$ vector and the vector $\hat{X}_{T+1}-X_{T}$ (with $\hat{X}_{T+1}$ standing for $\hat{X}_{T+1}^{-}$or $\hat{X}_{T+1}^{+}$). With the simulation of the Lorenz system described above, we achieve $92.8 \%$ accuracy, 
suggesting that this method of discrimination might be reasonable. However, we obtain only $67.3 \%$ accuracy with the logistic map. This is quite intuitive as the Lorenz system is "much less chaotic" than the logistic map (in the sense that its LLEs are "less often" positive and typically smaller than those of the logistic map; we elaborate on such distinctions of chaoticity in a companion paper) and, hence, is better behaved. Thus, with our rule of thumb, we achieve accuracy gains which are close to those obtained with best-candidate predictor on the Lorenz system. However, in the case of the logistic map (and highly chaotic systems, in general) our selector still needs refining. In another companion paper, we propose specific methods to improve upon the above rule of thumb to discriminate between candidates and, ultimately, yield better prediction results.

\section{References}

[1] Shintani M and Linton O. Nonparametric neural network estimation of Lyapunov exponents and a direct test for chaos, 2004; 120: 1-33.

[2] Guégan D and Mercier L. Stochastic and chaotic dynamics in highfrequency financial data. Signal Processing and Prediction, Prochazka Eds, 1998; 365-372.

[3] Doerner R, Hübinger B, Martienssen W, Grossman S and Thomae S, Predictability Portraits for Chaotic Motions, Chaos, Solitons and Fractals, $1991 ; 1: 553-571$.

[4] Rinaldi S, Casagrandi R and Gragnani A, Reduced order models for the prediction of the time of occurrence of extreme episodes, Chaos, Solitons and Fractals, 2001;12: 313-320. 
[5] Yousefi S, Weinreich I and Reinarz D, Wavelet-based prediction of oil prices, Chaos, Solitons and Fractals, 2005; 25: 265-275.

[6] Yang HY, Ye H, Wang G, Khan J and Hu T, Fuzzy neural very-short-term load forecasting based on chaotic dynamics reconstruction, Chaos, Solitons and Fractals, 2006;29: 462-469.

[7] Chian ACL, Rempel, EL and Rogers C, Complex economic dynamics: Chaotic saddle, crisis and intermittency, Chaos, Solitons and Fractals, 2006; 29: 1194-1128.

[8] Guerra FA and Coelho LS, Multi-step ahead nonlinear identification of Lorenz's chaotic system using radial basis neural network with learning by clustering and particle swarm optimization, Chaos, Solitons and Fractals, 2009; 35:967-979.

[9] Farmer JD and Sidorowich JJ. Predicting chaotic time series. Physical Review Letters, 1987; 59: $845-848$.

[10] Ziehmann C, Smith LA and Kurths J. Localized Lyapunov exponents and the prediction of predictability. Physics Letters A, 2000; 271: 237-251.

[11] Guégan D. Les Chaos en Finance: Approche Statistique. Economica Eds.; 2003, 420 pp.

[12] Abarbanel HDI. Local and global Lyapunov exponents on a strange attractor. Nonlinear Modeling and Forecasting, SFI Studies in the Science of Complexity, Casdagli M and Eubank S Eds, Addison-Wesley, 1992; Proc. Vol. XII: 229-247.

[13] Wolff RCL. Local Lyapunov exponents: looking closely at chaos. Journal of the Royal Statistical Society B, 1992; 54: 353 - 371. 
[14] Bailey B. Local Lyapunov exponents: predictability depends on where you are. Nonlinear Dynamics and Economics, Kirman et al. Eds, 1997.

[15] Eckmann JP and Ruelle D. Ergodic theory of chaos and strange attractors. Review of Modern Physics, 1985; 57: 615-656.

[16] Takens F. Estimation of dimension and order of time series. Progress in Nonlinear Differential Equations and their Applications, 1996; 19: 405-422.

[17] Takens F. Detecting strange attractors in turbulence. Lecture Notes in Mathematics, 1981; 898: 366-381.

[18] Lorenz EN. Deterministic non-periodic flow. Journal of Atmospheric Science, 1963; 20: 130-141.

[19] May RM. Simple mathematical models with very complicated dynamics. Nature, 1976; 261: 459-467. 